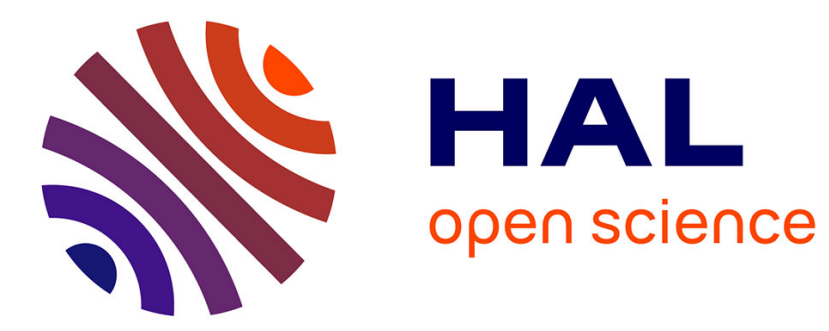

\title{
La controverse entre organicisme et vitalisme: étude de sociologie des sciences \\ Dominique Raynaud
}

\section{To cite this version:}

Dominique Raynaud. La controverse entre organicisme et vitalisme: étude de sociologie des sciences. Revue française de sociologie, 1998, 39 (4), pp.721-750. halshs-00005912

\section{HAL Id: halshs-00005912 \\ https://shs.hal.science/halshs-00005912}

Submitted on 20 Nov 2005

HAL is a multi-disciplinary open access archive for the deposit and dissemination of scientific research documents, whether they are published or not. The documents may come from teaching and research institutions in France or abroad, or from public or private research centers.
L'archive ouverte pluridisciplinaire HAL, est destinée au dépôt et à la diffusion de documents scientifiques de niveau recherche, publiés ou non, émanant des établissements d'enseignement et de recherche français ou étrangers, des laboratoires publics ou privés. 
Slightly revised for Revue française de Sociologie, 1998, 39 (4), pp. 721-750.

\title{
La controverse entre organicisme et vitalisme: Etude de sociologie des sciences
}

\author{
Dominique Raynaud*
}

Résumé. L'analyse de la controverse qui éclata entre les écoles de médecine de Paris et de Montpellier (18171852) montre que des intérêts professionnels, mais aussi des facteurs philosophiques et politiques conditionnèrent la crispation de l'école de Montpellier sur ses positions doctrinales. Mais ces facteurs n'expliquent pas le contenu des théories. Le règlement de la controverse fut étranger à une négociation sociale de la vérité, et tourna à la faveur ceux qui eurent la productivité scientifique la plus élevée. Cette controverse, qui échappe aux principes du programme relativiste en sociologie des sciences montre que certaines études peuvent s'être méprises sur le sens d'une détermination sociale des contenus scientifiques.

Mots-clefs. Controverse, médecine, organicisme, vitalisme, relativisme.

Summary. The study of the dispute that broke out between the medical schools of Paris and Montpellier (1817-1852) shows that professional interests, but also philosophical and political factors drew the fitgets of Montpellier's school about its doctrine. But these factors are inable to explain the scientific contents. Further, the closure of the controversy did not result from a social negotiation of truth. It simply favoured the scholars who had major scientific productivity. This dispute so contradicts the principles of the relativistic program in the field of the sociology of scientific knowledge, and shows that some studies could have misunderstood the sense of a social determination of scientific contents.

Keywords. Controversy, medicine, organicism, vitalism, relativism.

\section{Position du problème}

L'étude des controverses scientifiques est désormais devenue un sujet privilégié de la nouvelle sociologie des sciences, dont l'un des axes est d'expliquer comment se font et se défont les connaissances. En rupture avec l'approche mertonienne, centrée sur l'analyse de l'éthos scientifique et du fonctionnement de la science, ce courant envisage les contenus scientifiques comme objet d'étude sociologique, et non plus comme l'objet exclusif de l'épistémologie et de l'histoire des sciences.

\footnotetext{
*Université Pierre-Mendès-France, BP 47, 38040 Grenoble cedex 9 ; dominique.raynaud@upmf-grenoble.fr.
} 
Bloor (1976) et Collins (1981) ont défini des programmes d'analyse des contenus scientifiques dits « relativistes », parce qu'ils postulent qu'une théorie ne s'impose pas par adéquation intrinsèque au réel, mais à la suite d'une négociation sociale de la vérité. Les préceptes de l'« Empirical programme of relativism » de Collins (1981) illustrent parfaitement cette position:

1) Il faudrait montrer la flexibilité d'interprétation des théories car, la nature n'étant pas univoque, l'accord sur une théorie résulterait plutôt d'un consensus social.

2) Il faudrait décrire les procédures de construction du consensus qui sont notamment responsables du règlement des controverses scientifiques.

3) Il faudrait enfin envisager la relation entre ces procédures de limitation de la flexibilité interprétative et les structures politiques et sociales au temps où s'affrontent ces théories.

Le troisième point de ce programme assouplit significativement le principe de causalité du programme fort de Bloor. En effet, celui-ci demandait d'« expliquer le contenu et la nature mêmes de la connaissance scientifique » sous le régime de la «causalité efficiente » (Bloor, 1976: 1, 11-13). Le programme de Collins n'exige plus une détermination aussi stricte des contenus. En dépit de cette atténuation, les programmes de Bloor et de Collins émanent d'un même canevas, car ils supposent l'un et l'autre que la vérité est locale: il n'existerait que des ethnovérités. Cette position de fond conduit à se focaliser sur une question sociologique importante: comment rendre compte de la stabilisation des énoncés? Si l'on applique cette idée au sujet des disputes scientifiques, le règlement d'une controverse devrait être interprété comme une suite ininterrompue de négociations complexes et d'accumulation de dissymétries entre deux théories rivales, étant entendu que les éléments mobilisés par les pro et contra ne seraient pas seulement des arguments rationnels, mais aussi des convictions extra-scientifiques. Ce programme a été suivi, de manière plus ou moins nuancée, par un certain nombre de sociologues (Latour, 1989; Callon \& Latour, 1991; Collins \& Pinch, 1994, etc.) Nous entendrons ici par « relativisme », toute étude des contenus scientifiques adhérant au canevas général proposé par Bloor et Collins. Cette étiquette est évidemment restrictive: elle ne s'intéresse pas aux aspects généraux du relativisme qui pourraient apparaître au-dehors des questions de sociologie des sciences. Elle n'entend pas sous-estimer les nuances d'appréciation qui existent entre chacun des tenants du relativisme.

La thèse générale d'une détermination sociale des contenus scientifiques est loin de faire l'unanimité parmi les sociologues. Les critiques du programme relativiste se situent en général 
dans le sillage de la sociologie mertonienne et de l'épistémologie de Popper et de Lakatos. Le fondement de cette critique est que, entre deux théories rivales, celle qui l'emporte au terme de la controverse est celle qui présente un meilleur degré de correspondance au réel. Cette thèse est en désaccord formel avec le premier point du programme de Collins (1981) selon lequel: 1) la nature serait ouverte à des interprétations divergentes; 2) une théorie serait un artefact fragile reposant sur un consensus constamment révisable ${ }^{1}$. Du point de vue rationaliste, la vérité n'est pas un objet de négociation. Par des attaques répétées, Isambert $(1981,1994)$ s'est attaché à stigmatiser les contradictions internes de l'approche relativiste, et à montrer que l'unité des nouveaux sociologues des sciences tient davantage d'une « coalition antimertonienne » que d'une réelle unité théorique (1994: 57-65). Isambert ne nie pas pour autant que les contenus puissent être un objet de la sociologie, mais il conviendrait selon lui d'en entreprendre l'étude sans adhérer à un relativisme étroit dont les accents métaphysiques et conventionnalistes sont parfois très accusés. De même qu'on peut réunir certaines positions autour des principes généraux du relativisme, il est possible de nommer « rationalisme » — dans un sens tout aussi restrictif - toute approche se refusant à considérer les contenus scientifiques comme l'émanation d'un état, toujours variable et fluctuant, de la société. Cette étiquette n'entend pas forcer l'unité des représentants du rationalisme. Le collectif dirigé par Boudon et Clavelin (1994) témoigne lui aussi, à bien des égards, de nuances d'appréciation. Mais les auteurs s'interrogent tous sur la pertinence qu'il y a à soutenir la thèse d'une négociation sociale de la vérité.

Il me semble significatif que: 1) la plupart des questions posées par les rationalistes concernent le sens que l'on doit prêter à l'expression « détermination des contenus scientifiques » souvent utilisée par les relativistes; 2) l'assouplissement des positions relativistes, de Bloor à Collins, concerne précisément la nature de cette détermination. Nous touchons-là sans doute au coeur du débat rationalisme-relativisme. S'agit-il de détermination causale, d'influence extérieure, de conditionnement ou de simple correspondance? La nature de ce lien gagnerait beaucoup à être précisé.

\footnotetext{
${ }^{1}$ Dans La vie de laboratoire, Latour et Woolgar (1979) prétendent ainsi se démarquer de l'approche classique en insi stant sur l'aspect construit de la science, or: 1) cette position est fort ancienne en sociologie et en histoire des sciences, 2) et ils en tirent, par une sorte de paralogisme, l'idée que la réalité extérieure correspondant aux énoncés scientifiques n'existe pas: il n'existerait que des artefacts.
} 
Cet article a pour objet de réévaluer les arguments relativistes et rationalistes à partir de l'analyse concrète d'une controverse qui agita la médecine française du XIXe siècle, et dont les foyers respectifs furent situés à Paris et à Montpellier. Cette controverse, qui culmina de 1817 à 1852², opposa deux doctrines fortement divergentes: l' organicisme, soutenu à Paris par Corvisart (1755-1821), Broussais (1772-1838), Laënnec (1781-1826), Magendie (1783-1855) puis Claude Bernard (1813-1878); le vitalisme défendu par les disciples de Barthez (1734-1806) à Montpellier, Dumas (1765-1813), Bérard (1789-1829), Alquié (1812-1864) et surtout Lordat (1773-1870). Ces positions suscitèrent une adhésion souvent tranchée, mais on doit reconnaître que certains savants, comme Bichat (1771-1802) ou Dugès (1797-1838), s'essayèrent aussi à concilier ces doctrines antagonistes. Cette controverse peut intéresser la sociologie des sciences pour plusieurs raisons:

1) Sur le plan local, l'histoire de la faculté de médecine de Montpellier montre que le désaccord dépassa rapidement les seuls enjeux de la connaissance médicale, pour prendre le tour d'une polémique où les arguments politiques, métaphysiques, les intérêts professionnels et les jalousies personnelles ne furent pas écartés. Un stock aussi riche d'ingrédients extra-scientifiques est à même de révéler si des facteurs étrangers ont contribué au règlement de la controverse, et dans quelle mesure. Il peut en outre montrer si les positions scientifiques ont accusé des fluctuations et si la vérité a été l'objet d'une négociation.

2) Sur le plan international, l'histoire de la médecine du XIXe siècle montre que l'apogée de cette controverse coïncide avec le plus haut niveau de productivité scientifique médicale jamais atteint par la France. Ben-David (1994), utilisant les données corrigées de Garrison (1929), établit que, dans la période 1800-1830, les découvertes médicales françaises ont supplanté les découvertes anglaises, allemandes et américaines (Tableau 1).

\footnotetext{
${ }^{2}$ La controverse s'étend en amont et en aval de ces dates, mais les rapports entre les deux écoles n'ont jamais été aussi tendus que dans la période qui va de l'Examen des doctrines médicales de Broussais (1817) à la Doctrine anthropologique de Montpellier de Lordat (1852). Dans l'intervalle, on a publié huit livres dont le titre fait directement référence à la doctrine de Montpellier: 1818, 1819, 1819, 1830, 1845, 1846, 1851 et 1852. Le barycentre de ces dates est 1835. Précisons enfin qu'il fallut attendre la parution de l'Introduction à l'étude de la médecine expérimentale de Bernard (1865) pour que le leadership de la médecine parisienne soit définitivement confirmé.
} 


\begin{tabular}{lrrrrr} 
Décennie & France & G.B. & Allemagne & U.S.A. & Monde \\
\hline $1800-1809$ & 9 & 8 & 5 & 2 & 27 \\
$1810-1819$ & 19 & 14 & 6 & 3 & 47 \\
$1820-1829$ & 26 & 12 & 12 & 1 & 57 \\
$1830-1839$ & 18 & 20 & 25 & 4 & 71 \\
$1840-1849$ & 13 & 14 & 28 & 6 & 68
\end{tabular}

Tableau 1: Nombre de découvertes médicales par pays

L'Allemagne devait ensuite ravir la première place à la France vers 1840, avant de la perdre à son tour devant les U.S.A. à partir de 1910. Cette coïncidence laisse entendre que la controverse entre les écoles de médecine de Paris et de Montpellier est peut être intimement liée avec le niveau de productivité atteint par la France au début du XIXe siècle.

3) Si les hypothèses relativistes sont aisément reconstructibles sur la base des témoignages et des échanges connus entre les médecins des deux écoles, les études de sociologie des sciences de Ben-David (1994) offrent un cadre pour développer des hypothèses rationalistes alternatives. Joseph Ben-David s'est en effet livré à une analyse comparée des organisations scientifiques et médicales de différents pays au XIXe siècle. Il montre notamment que la différence de productivité scientifique ne peut pas être interprétée comme une conséquence directe, ni de la richesse comparée des nations, ni de la circulation différentielle des connaissances dans ces pays.

On a donné un grand nombre d'explications du leadership de l'Allemagne sur la France à partir de 1850 (aptitude à identifier de nouveaux champs de recherche; meilleure organisation de l'Université; haut niveau de spécialisation de l'enseignement; existence d'institutions nombreuses facilitant la mobilité des savants). Ben-David pondère l'importance de ces facteurs, en montrant que la médecine expérimentale allemande se développa en calquant partiellement le modèle français; que l'Habilitationsschriften, en dépit de sa spécificité, était souvent un travail de recherche de niveau comparable à l'agrégation française (1994: 115). Selon lui, seul un facteur institutionnel explique ce leadership. Il résulterait de l'autonomie des universités allemandes, par opposition à la centralisation de la médecine française, qui n'était plus à même de répondre aux exigences de la recherche médicale vers 1850. À long terme, le centralisme impulsé par les Montagnards de la Convention et renforcé par le Premier Consul en l'an VIII (1799) aurait freiné le développement 
autonome des chaires de spécialités ${ }^{3}$ contraignant ainsi les savants à faire de la recherche méd icale une activité secondaire, souvent dilettante. Cependant que le centralisme français empêchait la multiplication des institutions de recherche, le gouvernement prussien procédait à la création répétée de nouveaux laboratoires (Ben-David, 1994: 123). La mobilité des savants français au sein de l'organisation scientifique en aurait été réduite d'autant, et l'émulation brisée par le mandarinat. Ce portrait, exact dans ses grandes lignes, ne tient pas compte de la diversité de situation des universités françaises. Il demanderait - dans le cadre de cette étude - à être nuancé par une analyse des situations des écoles de Paris et de Montpellier.

\section{Le rayonnement de l'école de Montpellier}

Le prestige de l'école de médecine de Montpellier, à la fin du XVIIIe siècle et au début du XIXe siècle, peut être estimé qualitativement par un certain nombre de témoignages historiques. Mais il peut être aussi apprécié au travers d'indices plus tangibles, au nombre desquels on doit placer les effectifs étudiants et les facilités d'accès au savoir.

\subsection{Les effectifs étudiants}

Les travaux de Julia \& Revel (1989) permettent de constater la dissymétrie qu'accusait, sous l'Ancien Régime, la population étudiante des facultés de Montpellier et de Paris. Les premières inscriptions (ou matricules) prises auprès de l'une ou de l'autre témoignent de la nette prééminence de Montpellier (Tableau 2).

\begin{tabular}{lrr} 
Université & période & étudiants \\
\hline Montpellier & $1760-1789$ & 71,2 \\
Paris & $1753-1774$ & 45,5 \\
Strasbourg & $1760-1789$ & 35,7
\end{tabular}

Tableau 2: Nombre moyen de premières inscriptions (1753-1789)

\footnotetext{
${ }^{3}$ Le nombre restreint de chaires provoquait des nominations « en chaîne ». Par exemple, Glaude Bernard ne céda la chaire de Physiologie expérimentale de la Sorbonne à Paul Bert, que lorsqu'il put occuper celle de Magendie au Collège de France (1855).
} 
Les effectifs totaux (matricule, baccalauréat, licence et doctorat) peuvent être reconstruits à partir des registres et des droits (Julia \& Revel, 1989: 472-473, 478-479). Ils confirment le leadership de l'école de Montpellier: dans la période 1780-1789, l'effectif annuel moyen fut 268,4 à Montpellier, 106,6 à Paris.

L'enquête lancée en prairial an XIII (juin 1805) par le Ministère de l'Intérieur, pour connaître les effectifs des professions de santé, conforte, par une autre voie, cette image de l'école de Montpellier. Le nombre des médecins en exercice en France en 1803-1806, qui avaient pris leurs grades avant 1794, accuse en effet une dissymétrie encore plus frappante (Tableau 3).

\begin{tabular}{lrr} 
Université & effectif & \% \\
\hline Montpellier & 1101 & 45,9 \\
Toulouse & 226 & 9,4 \\
Caen & 195 & 8,1 \\
Reims & 156 & 6,5 \\
Besançon & 122 & 5,1 \\
Douai & 96 & 4,0 \\
Nancy & 95 & 3,9 \\
Paris & 72 & 3,0 \\
Strasbourg & 38 & 1,6 \\
France & 2398 & 100,0
\end{tabular}

Tableau 3: Origine des médecins français (1803-1806)

Julia (1992) a très justement insisté pour que l'on pondère cette représentation par deux remarques visant les effectifs de Paris et de Strasbourg. Tout d'abord, la faculté de Strasbourg délivrait beaucoup de diplômes à des étudiants étrangers (Suisses et Allemands, principalement) qui ne se destinaient pas à l'exercice de la médecine en France. Ensuite, les droits perçus par la faculté de Paris étaient si élevés (4300 livres en moyenne) que l'accès en était réservé aux étudiants les plus fortunés (Julia \& Revel, 1989: 281). Les autres effectuaient la quasi-totalité du cursus à Paris avant de s'inscrire dans une université moins prestigieuse pour obtenir les grades. Cette inscription s'effectuait généralement dans les universités les plus proches (Reims, Douai et Caen). Mais, 
même dans ces conditions, les grades délivrés à Montpellier sont deux fois plus nombreux que les grades dispensés par les quatre universités conjointes (Tableau 4).

\begin{tabular}{lrr} 
Université & effectif & $\%$ \\
\hline Montpellier & 1101 & 45,9 \\
Paris-Reims-Douai-Caen & 519 & 21,6 \\
Autres & 778 & 32,5 \\
France & 2398 & 100,0
\end{tabular}

Tableau 4: Effectifs des docteurs de Montpellier et Paris

Conclusion: avant la suppression des facultés votée par la Convention en l'an I (1792), un médecin sur deux était issu de la faculté de Montpellier, un médecin sur cinq venait de Paris.

\subsection{Les facilités d'accès au savoir}

La première hypothèse qui vient à l'esprit pour expliquer ce niveau de prestige de l'école de Montpellier est qu'elle aurait su conserver son attractivité depuis la fondation de l'université médiévale. C'est oublier les grandes crises que l'université de médecine de Montpellier dût affronter au cours des siècles. On trouve, dans les années précédant immédiatement la controverse étudiée, des raisons plus sérieuses pour expliquer ce rayonnement. Il apparaît en effet que Montpellier sut se doter d'une bibliothèque médicale de premier plan, facilitant ainsi les conditions d'accès au savoir des professeurs et des étudiants. Gabriel Prunelle, dont la nomination comme bibliothécaire de l'école de santé de Montpellier fut approuvée par le Ministère de l'Intérieur le 28 vendémiaire an VI (1797), en sera la cheville ouvrière. Rencontrant, à partir de l'an XII (1803), quelques difficultés à extraire des dépôts nationaux les collections qui valaient d'être intégrées à la bibliothèque, le citoyen Prunelle demande l'appui de Jean-Antoine Chaptal, alors Ministre de l'Intérieur, lequel avait été reçu docteur en médecine à Montpellier en 1777. Soutenu par plusieurs membres de l'Académie des Inscriptions et Belles-Lettres, Chaptal nomme alors Prunelle « commissaire du Gouvernement chargé de l'inspection des bibliothèques et dépôts littéraires » (Vidal, 1958: 90). Cette autorité lui permettra de prendre dans les dépôts du Mans, de Chartres, de Dijon, d'Auxerre et de Troyes. 
L'étude de la correspondance de 1802 à 1807 (Vidal, 1958) entre Prunelle et René, directeur de l'école de santé, témoigne de l'étendue de ses découvertes. Le citoyen Prunelle obtient le droit de procéder à des ventes de manière à acquérir de nouvelles bibliothèques comme celle d'Albani ou de l'Héritier. Il écrit vouloir « procurer à la bibliothèque des livres modernes de médecine dont nous sommes absolument dépourvus; J'ai demandé 10000 Francs pour cet objet », à tel point qu'à Montpellier les locaux vont rapidement faire défaut pour accueillir les collections. Prunelle ne se contente pas de prospecter en France. Il prend des contacts à Hanovre, et suggère à René de contacter les facultés de Leyde, Göttingen et Iéna, pour mettre en place un échange des thèses (Vidal, 1958: 91). De plus, en 1806, Paul-Joseph Barthez, professeur à Montpellier et médecin consultant de l'Empereur, décède et lègue tous ses livres à l'école de médecine de Montpellier « pour accroître sa bibliothèque publique » de quelques 5000 volumes. Après avoir prospecté de nombreux dépôts en France et à l'étranger, Gabriel Prunelle rentre à Montpellier où il est nommé professeur de Médecine et d'Histoire de la Médecine, par un décret de Napoléon du 10 novembre 1807. On trouve, dans son Cours de propédeutique médicale, l'idée que l'accès au savoir est l'une des conditions du progrès des connaissances: «L'instrument de travail fondamental, c'est une bibliothèque dans laquelle on trouve, non pas des manuscrits précieux, mais une collection de tous les ouvrages de prix que la fortune ordinaire d'un particulier ne lui permet pas d'acquérir, et de tous les livres manuels qui peuvent être à l'usage des étudiants... Messieurs les Elèves, les études que vous avez faites jusqu'à présent ne sont en quelque sorte qu'une préparation à celles qui vont occuper votre vie entière... » (Des études du médecin, de leurs connexions et de leur méthodologie, 17 novembre 1815). Cette idée avait déjà été exprimée par Charles-Louis Dumas, professeur de médecine à Montpellier, alors que Prunelle débutait à peine la prospection des dépôts (20 germinal an XII): « Il faut enrichir les bibliothèques de tous les livres qui attestent les progrès de la médecine... et lui préparer ainsi de plus grandes ressources à son perfectionnement futur... Quelque esprit de génie [allusion à Barthez] envisage le progrès de l'esprit humain comme sans borne... » (Discours sur les progrès futurs de la science de l'homme).

On déduit sans peine de l'étude de la population étudiante et des conditions d'accès au savoir que l'école de médecine de Montpellier avait, dans les années 1810, les ressources nécessaires et suffisantes pour engager un bras de fer avec Paris. Elle l'avait supplantée par ses effectifs et venait de se doter d'un fonds susceptible de contribuer au développement de sa doctrine médicale. 


\section{Les aspects de la controverse}

Il convient de décrire les événements par lesquels s'opposèrent les deux écoles de médecine de Paris et de Montpellier. Lorsqu'on prend le parti d'étudier une controverse entre deux savants isolés (Pasteur et Pouchet), il est relativement aisé de retracer l'histoire complète des débats. En revanche, l'étude d'une controverse entre deux groupes de chercheurs, préparée par des rivalités professionnelles anciennes ${ }^{4}$, ne permet pas de prétendre à l'exhaustivité. On peut toutefois donner quelques échantillons qui permettent de prendre la mesure de l'opposition, à la fois sur le plan scientifique et sur le plan humain.

\subsection{Les aspects scientifiques}

Bérard fit paraître en 1819, des Observations cliniques pour servir de preuve à la doctrine médicale de l'école de Montpellier. On y trouve la description des complications d'un cas de colique néphrétique. Le patient, un magistrat de 60 ans, avait été opéré par la taille le 24 avril 1819, ce qui permit l'extraction d'un « calcul ovoïde de six onces ». Au huitième jour après l'opération, le patient souffrit de délire. Le neuvième jour, il évacua spontanément une once et demie de sang par la plaie. Le dixième jour, la plaie libéra à nouveau une grande quantité de sang rutilant. Voici les signes cliniques sur lesquels Bérard fonde son diagnostic:

« Le déclin de l'avant-veille, l'hémorrhagie légère de la veille, sur-tout la coïncidence de l'heure où ces événemens étaient survenus, les conditions vernales très-prononcées de la saison, la co-existence d'un assez grand nombre de fièvres intermittententes... se réunissaient pour faire croire à l'existence d'une fièvre intermittente quotidienne dont l'hémorrhagie n'avait été que le symptôme. » (1819: §1).

\footnotetext{
${ }^{4}$ Notons que cette controverse entre les écoles de Paris et de Montpellier avait eu des antécédents dès le XVIIe siècle, lors de la fameuse « Guerre de l'Antimoine ». Elle avait opposé Simon Courtaud et Isaac Carquet aux Parisiens Gui Patin, Jean Riolan et Charles Guillemeau. Les faux utilisés par le doyen Courtaud dans son apologie de l'école de Montpellier en 1645 déclenchèrent les foudres des Parisiens. Carquet prit la défense de Courtaud dans une Seconde apologie (1653). Les Parisiens réagirent par une Responsio pro se ipso ad alteram Apologiam impudentissimi et importunissimi Curti Monspelcaniscellarii (1654), et par une Defensio altera, adversus impias impuras et impudentes tum in se, tum in Principem Medicinae Scholam Parisiensem, Anonymi Copreae calimnias ac contumelias (1655). L'affaire prit un tour d'une rare violence (Dulieu, 1983: 609).
} 
Bérard venait de découvrir une « affection générale » dirigée par un « génie périodique pernicieux » (sic), qui apportait une preuve évidente en faveur de la doctrine de Montpellier, laquelle supposait l'existence de maladies générales (non-locales) n'ayant pas de causes organiques. Il va sans dire que le cas présenté par Bérard aurait été interprété très différemment par les médecins parisiens. La coïncidence des heures, pas plus que l'influence du printemps, n'auraient été prises en compte par eux pour établir leur diagnostic. Ce que le vitalisme accueillait donc sans difficulté, l'organicisme le rejetait tout aussi catégoriquement. Comment attribuer les complications de cette opération à une altération de la force vitale, non-localisable dans l'organisme et immatérielle de surcroît? Cet exemple montre les tendances opposées suivies par Montpellier et Paris. Le vitalisme tendait à unifier le siège des maladies en différenciant les affections (cf. la classification des « éléments morbides » ou des fièvres) alors que l'organicisme unifiait les affections en différenciant leur point d'application. Voilà un exemple, parmi tant d'autre, qui indique la différence appréciable de conception entre les deux écoles. Celle-ci devra être examinée en détail.

\subsection{Les aspects humains}

Une controverse peut se limiter à une opposition d'arguments rationnels, mais elle peut aussi, lorsque les chercheurs viennent à court d'arguments, déplacer les rivalités scientifiques sur le plan personnel. Au début, les Parisiens se contentèrent d'émettre des doutes sur le vitalisme en évitant toute polémique. Bichat dit: «Qu'est l'observation si l'on ignore le siège du mal? » Et Magendie signale dans les textes de Montpelliérains « certains mots inintelligibles, tels que la Force ou Principe vital » (1825: viii). Le conflit fut exacerbé par la publication des Fragments de philosophie de Hamilton, dans laquelle le traducteur, un certain Peisse, fit de sévères remontrances aux vitalistes: «L'école de Montpellier s'est tellement attachée à la Métaphysique qu'elle en a souvent oublié la Médecine ». Il s'ensuivit une polémique qui prit la forme d'un feuilleton. On peut en suivre les épisodes dans la Gazette médicale de Paris (1841-1843). Lordat répondit par une Apologie de l'école médicale de Montpellier (1841). Après avoir dressé la liste des sous-entendus de son contradicteur, il les attaque un à un, non sans ironie:

« [L'école de Montpellier] sait bien que des malfaiteurs veulent la noyer; elle a tremblé qu'ils n'eussent surpris votre honorable et imposante signature dans de calomnieux et méchants actes de notoriété de rage... En contractant 
autant que je le puis les reproches que vous faites à notre Ecole, je crois pouvoir les réunir sous les chefs suivants: $1^{\circ}$ Elle a l'esprit et les tendances de l'Ecole platonicienne, et par conséquent elle doit être plus contemplative qu'active. $2^{\circ}$ Elle doit être paresseuse puisque l'esprit platonicien est paresseux. $3^{\circ}$ Par conséquent, elle est immobile. $4^{\circ}$ Elle forme des Philosophes-Médecins plutôt que des Médecins-Philosophes. $5^{\circ}$ Elle est persuadée que la Science médicale est finie et n'a plus rien à faire. $6^{\circ}$ Partout elle est stérile... Nous savons très-bien que l'excision des amygdales et du voile du palais ne peut rien faire pour la guérison immédiate du bégaiement... » $(1842: 10,38)$.

Peisse, bien sur, refusa de participer à la « négociation secrète » (1842: 72) que lui proposait Jacques Lordat. Piqué au vif, il entreprit même d'expliciter davantage ses objections:

« Ge sont de simples croyances en droit hors de la Science, mais qui la simulent. De-là, naît pour la Médecine, une illusion tout-à-fait analogue dans son principe et dans ses résultats à celle qu'a signalé le règne des Doctrines occultes... Montpellier s'est trouvée ainsi peu à peu dans une sorte d'isolement intellectuel. Au lieu de suivre ces grands mouvements qui se font autour d'elle ou du moins d'y participer, elle se contente de les observer de loin et de haut; elle ne les considère que... comme une tempête passagère au milieu de laquelle sa Doctrine, semblable à la barque de SaintPierre qui ne doit pas périr, peut bien être ballottée, mais non submergée. » (1842).

Un disciple de Lordat, Kühnholtz, devait poursuivre cette polémique en mettant en cause plus directement le traducteur et ses perfides conseillers parisiens:

« Il s'ensuit que ses études de médecine ont été faites, un peu comme le voyage de M. Maistre, autour de sa chambre... [La physiologie expérimentale], quoiqu'en dise M. Magendie, mérite bien plus le titre de Roman de la Médecine... La physiologie médicale de Paris amène directement à l'adoption de beaux Principes que M. J. A. X., Membre de l'Académie royale de Médecine, a eu l'impudeur de proclamer et de publier dans son livre intitulé: De l'épicurisme et de ses applications... Voici à quoi se réduisent les Principes de l'auteur... Il n'y a point de Dieu. Il n'y a point d'âme... » [Suit un commentaire dans lequel Kühnholtz se dit choqué par ce témoignage d'un " cynisme révoltant ».] « N'en veuillons pas trop néanmoins à l'Ecole rivale et peut-être ennemie de la Nôtre. Nous sommes de ceux qui pensent que les aînés de bonne famille doivent des égards à leurs puînés... » (1843: 22, 45, 65, 71).

Les rivalités scientifiques cédèrent donc rapidement le pas aux sarcasmes pleins de mépris et d'arrogance. Les fâcheries ne s'arrêtèrent pas là. En 1852, Barbaste répétait qu'« Il fallait aux novateurs [parisiens] une philosophie un peu crasse, un peu épaisse, selon le goût de Madame de Sévigné »(1852: 1). Le 10 juillet 1860, Chrestien adressait une lettre à Rouland, Ministre de l'Instruction publique et des Cultes. Il y exposait l'antagonisme irréductible entre les deux écoles sous forme 
d'une vibrante tragédie antique. Paris y jouait Calchas; Montpellier Iphigénie... Ce type d'ingrédients affectifs et passionnels laisse facilement suspecter le rôle de facteurs extra-scientifiques dans la controverse.

\section{Interprétation sociologique}

Il reste à interpréter pourquoi cet échange polémique se termina à l'avantage de la médecine parisienne. L'échec de Montpellier à imposer le vitalisme a toujours suscité de nombreuses questions. Est-il dû à une différence des méthodes? (expérimentale vs. spéculative), des doctrines? (réductionnisme vs. holisme), des a priori philosophiques? (matérialiste vs. spiritualiste). Le partage s'est-il opéré selon une différence de productivité scientifique? (forte vs. faible), des institutions? (extra-universitaire vs. universitaire). Y a-t-il eu, enfin, collusion d'intérêts professionnels? (centralisme vs. régionalisme) ou politiques? (démocratie vs. monarchie). On peut, bien sûr soupçonner le rôle de ces éléments, et toutes ces hypothèses méritent un examen approfondi.

L'analyse en facteurs que l'on doit à présent envisager présentera aux yeux de certains le défaut d'une clarification excessive de la réalité. Il est vrai que la séparation des facteurs institutionnels, professionnels et politiques, n'est jamais d'une parfaite netteté (l'organisation d'une institution scientifique émane toujours, au moins en partie, d'une volonté politique). Mais cette séparation des facteurs paraît incontournable. Même les plus purs relativistes, promoteurs du principe d'inséparabilité entre science et société, en font usage. Lécuyer et al. ont noté: « Les partisans du "programme fort" déclarent que la société et la science ne font qu'un; mais, dans leur pratique, ils distinguent clairement entre évolutions "internes" et évolutions "externes" à la science » (1988: 406). La critique ne touche pas que les travaux anglo-saxons réunis dans Les scientifiques et leurs alliés (Callon et Latour, 1985). Dans l'étude de la controverse entre Pasteur et Pouchet, par exemple, Latour s'empresse de réintroduire une démarcation entre la science et la société lorsqu'il entreprend de démontrer ce que doit l'asymétrie des deux savants à l'« idéologie » (1989: 437). Ce type d'analyse reste donc parfaitement légitime.

Reste à préciser l'ordre dans lequel nous allons procéder. Dans l'étude qu'il consacre à cette période mouvementée de l'école de Montpellier, Dulieu (1990) suspecte le rôle des intérêts profes- 
sionnels et des facteurs politiques dans cette controverse. Néanmoins, il assure que cette querelle concerna avant tout des points de doctrine médicale: « Deux écoles s'affronteront ainsi constamment: Paris et Montpellier. Disons tout de suite que les jalousies professionnelles y joueront un rôle mais aussi les questions politiques entretenues par les nombreux changements de régime que connut le XIXe siècle. Il n'en reste pas moins que l'esprit dans lequel on abordait l'étude de la médecine constituera l'élément essentiel de ces controverses. » (1990: 212). Cette position justifie que nous commencions l'analyse par les questions de méthode et de doctrine, en nous dirigeant progressivement vers l'examen des facteurs extra-scientifiques.

\subsection{Le facteur méthodologique}

L'un des enjeux de la controverse vitalisme vs. organicisme concernait les méthodes qui pouvaient être appliquées à l'étude de la médecine. La différence qui se fit jour entre les deux écoles peut être stigmatisée par l'opposition entre la méthode expérimentale et la méthode spéculative.

Les promoteurs de la médecine parisienne furent tous des cliniciens et des observateurs réputés. Corvisart, qui exerçait la clinique interne à l'hôpital de la Charité, rejetait toute théorie médicale et prétendait que son Essai sur les maladies et les lésions organiques du coeur constituait un « ouvrage de pure pratique, fondé sur l'observation et l'expérience » (1818: iii). Laënnec, qui avait débuté dans le service de Corvisart à la Charité, se défendait également d'adhérer à quelque théorie médicale que ce soit. L'expérience était pour lui une priorité. Ce n'est pas là une simple recommandation théorique: Laënnec fit 22 autopsies au cours des seuls mois de septembre-octobre 1822 (Ackerknecht, 1986 : 122). Il avait d'ailleurs adopté la devise: Ars medica tota in observationibus, et sa thèse se termine par ces mots significatifs: "Je n'appartiens ni aux Anciens, ni aux Modernes; je les suis tous les deux quand ils disent le vrai. Je crois en l'expérience fréquemment répétée. »(1804: 38).

La même orientation peut être remarquée chez Broussais, bien que celui-ci n'ait pas contribué, à proprement parler, aux débuts de la médecine expérimentale. Avant d'être nommé professeur à l'Hôpital du Val-de-Grâce (1814), Broussais s'était enrôlé comme chirurgien de la Marine. Médecin de l'armée des côtes de l'Océan et des campagnes napoléoniennes, il profita de ses multiples observations pour développer ses thèses localistes. Enfin, on se dispensera d'une étude détaillée de Magendie et de Claude Bernard, à qui l'on doit les progrès les plus significatifs dans le 
domaine de la physiologie et de la pathologie expérimentale/s (Ackerknecht, 1986: 161).

Sur ce point, Bichat doit être rapproché de l'école de Paris. Il avait ouvert un cours d'anatomie en 1797, où l'on pratiquait les dissections et les démonstrations physiologiques. Il aurait fait plus de 600 autopsies dans l'hiver de l'an X (1801). Ces observations sont à l'origine de ses découvertes sur la sensibilité (système sympathique) et la contractilité (système nerveux). Par ailleurs, l'approche de Bichat était souvent de nature expérimentale. Certaines de ses descriptions s'appuient sur des expériences de transfusion de sang artériel, d'autres sur l'absorption d'air au niveau de l'intestin (Ackerknecht, 1986). Comme le dit Dulieu: «Bichat, qui n'avait pas étudié à Montpellier mais dont le père en était docteur, ne niera pas l'existence de l'âme mais, celle-ci n'étant pas soumise à l'expérience, il la laissera de côté, préférant s'occuper de ce qu'il pouvait observer. » (1975: 213).

À l'opposé, les Montpelliérains furent peu enclins à pratiquer la médecine d'observation et la médecine expérimentale. Barthez, reçu docteur en médecine en 1753, enseigna sa vie durant une médecine théorique coupé de l'observation et la clinique. Il ne fit qu'une étude concrète portant sur la goutte (1802). Le cas de Dumas est plus nuancé puisqu'on lui doit une anatomie musculaire (1797), une étude des maladies chroniques (1812), mais surtout une Introduction à la science expérimentale (1800), dans laquelle il défend une approche inductive de la médecine, quoique d'une manière par trop théorique. Lordat présente un profil analogue à celui Dumas. Théoricien dogmatique, il conçut de justifier le bien fondé de la doctrine de Montpellier par un accolement tardif à la philosophie expérimentale inductive (1848, 1858). Mais c'est là une recommandation abstraite, qui ne s'est accompagnée d'aucune étude concrète en physiologie expérimentale. Bérard ne fut pas davantage impliqué dans les innovations médicales de son temps: il rédigea un essai sur la variole (1818) et quelques observations cliniques censées « servir de preuves » à la doctrine de Montpellier (1819). Seul Alexis Alquié, qui était chirurgien en chef de l'Hôtel-Dieu, s'attacha à étudier de problèmes précis de pathologie et de clinique chirurgicale, sans pour autant renier la doctrine de Montpellier.

On constate donc une dissymétrie accusée entre les Parisiens promoteurs de l'observation expérimentale, et les Montpelliérains qui échafaudèrent une « doctrine anthropologique » plus spéculative que descriptive. Certains ont d'ailleurs fait observer que plus les progrès de la physiologie expérimentale se confirmaient, plus « Montpellier s'arc-boutait sur la doctrine barthézienne 
» (Lavabre-Bertrand, 1992: 63). La tournure d'esprit expérimentaliste vs. spéculative est la première cause du désaccord entre les deux écoles.

\subsection{Le facteur doctrinal}

La controverse se répercuta plus fondamentalement sur les points de doctrine les plus directement connectés à ces méthodes. L'observation et l'expérimentation, parce qu'elles étaient incompatibles avec une approche globale du corps humain, jouèrent un rôle dans le développement des thèses localistes de Paris. Ces thèses - issues pour partie de la théorie de l'irritabilité de Haller et de Tommasini - remettaient en question la notion hippocratique de «maladie générale » selon laquelle toute affection locale prend son origine dans un déséquilibre général du corps. La thèse localiste sera d'autant plus vigoureusement affirmée que Paris compte alors un certain nombre de représentants de la doctrine animiste de Stahl, qui préconise, par une sorte d'extrapolation de l'hippocratisme, que l'âme, siège de toutes les maladies, doit veiller elle-même à son propre rétablissement. Selon le localisme, au contraire, toute affection résulte d'un dysfonctionnement organique précis. On reconnaitra ici la réplique médicale d'une thèse connue sous le nom de réductionnisme: Le corps n'est pas une totalité indivisible, mais la somme ordonnée d'organes et de fonctions. Les localistes ont appliqué cette thèse à de multiples sujets. Broussais, par exemple, soutient que les états de la conscience peuvent être réduits au produit de l'action de la «substance nerveuse interne » (1826). Corvisart ne fut pas un localiste stricto sensu, mais on observe chez lui le même souci de considérer l'homme comme un mécanisme, dont on peut observer l'une ou l'autre des parties. Le projet, Des localisations et des causes des maladies étudiées au moyen des signes diagnostiques et confirmées par l'autopsie, suffit à démarquer Corvisart de l'hippocratisme, Bien qu'en thérapie Corvisart eut une attitude nuancée. Son élève, Laënnec, raconte qu'il louait fréquemment l'approche hippocratique - passiviste - tout en recourant à des thérapeutiques actives comme la cautérisation en cas de phtisie (Ackerknecht, 1986: 173).

Alors que la méthode expérimentale était compatible avec la médecine organique d'inspiration réductionniste lancée par Corvisart et Broussais, la méthode spéculative des médecins de Montpellier exigeait une conception holiste de la médecine. Cette approche est suggérée par certains textes des promoteurs du vitalisme. Le 20 germinal an XII (1803), Dumas, professeur à Montpellier, dévoile la nature des spéculations de l'école qui cherche alors une médecine totale: 
«Pourquoi séparer la philosophie et la médecine? N'ont-elles pas l'une et l'autre pour objet de rassembler tous les rapports qui lient l'homme à la nature? (...) Le médecin se compose un fonds vaste, inépuisable en recevant le tribut de plusieurs sciences dont il devient l'heureux dépositaire. La destinée d'une multitude prodigieuse de connaissances repose donc entre ses mains... Barthez voit toutes sciences au service de la médecine et la médecine toute puissante au service de l'homme... » (Discours sur les progrès futurs de la Science de l'Homme).

Cette conception holiste pouvait — à quelques flottements sémantiques près — s'accommoder de recommandations prestigieuses. Elle était en effet partiellement cohérente avec les canons de l'ancienne médecine, et les vitalistes de Montpellier ne manquèrent pas de clamer, à chaque fois qu'ils étaient pris en défaut, leur attachement à la doctrine hippocratique ${ }^{5}$.

Mais ce n'était pas là l'hippocratisme des Sydenham, Barbeyrac, Corvisart ou Laënnec, qui voyaient dans le médecin de l'Antiquité le fondateur de la médecine d'observation. À l'opposé, Hippocrate était surtout vénéré à Montpellier comme auteur de la maxime: «Transporter la philosophie dans la médecine et la médecine dans la philosophie ». Cette lecture originale quoique congruente avec l'idée hippocratique d'une natura medicatrix régulant ses propres dysfonctionnements - transparaît dans les textes des Montpelliérains, depuis le Discours sur le génie d'Hippocrate de Barthez (1801) jusqu'aux essais de Lordat: Vicissitudes de l'anthropologie hippocratique (1856) et Rappel des principes doctrinaux de la constitution de l'homme énoncés par Hippocrate (1857). On y trouve toujours la marque d'une conception holiste de la médecine, que Barthez nommait « Science de l'Homme » (1778), et que Lordat appellera plus tard « Anthropologie » (1852). Pour Barthez et ses disciples, le « principe vital » est partout et agit en tous les endroits du corps. C'est une force diffuse qui garantit l'unité indivisible du corps humain. Alquié, par exemple, préconise encore une « étude de l'homme dans son entier, sans se laisser captiver par les manifestations locales » (Dulieu, 1990).

On distingue ici une deuxième opposition qui partage significativement les doctrines respectives des écoles de Paris et de Montpellier, même si l'on peut toujours identifier quelques divergences de vue entre deux membres d'une même école.

\footnotetext{
${ }^{5}$ Cette association vitalisme-hippocratisme connaît cependant un contre-exemple: Xavier Bichat, qui n'était pas un adversaire des thèses vitalistes, refusait la classification a capite ad calcem et l'hippocratisme tout entier.
} 


\subsection{Le facteur philosophique}

On a voulu lire dans cette controverse l'affrontement d'une philosophie matérialiste et d'une métaphysique spiritualiste. On touche ici à un point qui dépasse bien évidemment les seuls enjeux médicaux et concerne une conception plus générale des rapports de l'homme au réel.

Corvisart, qui se présentait clairement comme un adversaire des théories spéculatives, adopta néanmoins quelques unes des idées de Bichat sur l'activité cardiaque et l'inflammation. Mais comme le souligne Ackerknecht, en dépit de ces emprunts, « Corvisart n'était pas un vitaliste; pour lui l'homme n'était qu'une machine. » (1986: 111). La connaissance concrète des phénomènes vitaux valait mieux que toute spéculation sur l'origine de la vie. Ce trait est partagé par Broussais, bien qu'il ait pour sa part érigé cette attitude en système. Broussais fut, sa vie durant, mais plus encore après avoir accusé un recul d'audience à partir des années 1820, un ardent défenseur du matérialisme de Cabanis. Dans l'essai De l'irritation et de la folie (1826), il attaque les philosophies spiritualistes et éclectiques de l'époque, en prônant une médecine coupée de toute spéculation sur l'âme comme siège de la coordination des fonctions vitales.

La doctrine barthézienne s'oppose sur ce point à la doctrine parisienne, puisque le « principe vital » suppose bien une telle coordination. Pourtant Barthez n'était pas un adepte de l'animisme. À la fin du XVIIIe siècle, le vitalisme se présentait comme une doctrine conciliatrice de l'irritabilité défendue par Albert von Haller (1708-1777), William Cullen (1712-1790) et John Brown (1735-1788), et de l'animisme de Georg Ernst Stahl (1660-1734). Le «principe vital » devait donc se trouver à égales distances de l'âme et des phénomènes physico-chimiques (Dulieu, 1983). Une différence essentielle entre l'animisme et le vitalisme était que, contrairement à l'âme, le principe vital était de nature périssable. Par conséquent, comme l'a bien noté Lavabre-Bertrand (1992), l'opposition matérialisme vs. spiritualisme ne suffit pas à caractériser les termes de cette controverse. La doctrine de Barthez ne suppose pas les prolongements métaphysiques qu'on a voulu lui faire endosser. Deux éléments permettent de s'en assurer.

Premièrement, dans ses écrits théoriques, Barthez n'a jamais tenté de décrire le siège du « principe vital ». Il s'interdit toute conjecture sur l'origine et la nature de cette entité. Le principe vital résulte d'une synthèse abstraite des «petites vies » de Bordeu. Barthez montre qu'elles sont l'expression d'un principe unique qui les réunit, les coordonne, et qui a pour fonction de maintenir le corps en vie. 
Deuxièmement, les sociologues seront sensibles au fait que l'une des influences de la doctrine barthézienne s'est exercée sur l'oeuvre d'Auguste Comte, qui avait suivi ses cours en 1816-1817 à Montpellier, après son renvoi de l'Ecole polytechnique et avant de devenir le secrétaire de SaintSimon. Il y aurait quelque contradiction à vouloir forcer les liens entre la médecine parisienne et le positivisme comtien, en oblitérant par ailleurs tout rapport entre le système de Comte et celui de Barthez.

Le vitalisme s'oppose bien au réductionnisme des initiateurs de la médecine expérimentale, mais n'exige pas pour autant une métaphysique spiritualiste. La doctrine barthézienne reste une phénoménologie. Si l'on est en droit de parler de spiritualisme, ce n'est qu'à propos des successeurs Montpelliérains de Barthez. Le vitalisme adopté par Bérard, sous la forme d'une Doctrine des rapports du physique et du moral pour servir de fondement à la métaphysique (1823), dénote une articulation avec la thèse animiste. La collusion avec le spiritualisme sera plus explicite dans les écrits de Lordat. Celui-ci suppose, à côté des forces organiques, l'existence de forces inorganiques d'ordre supérieur qui composent l'âme humaine. Cette combinaison de l'âme pensante et de la force vitale est à l'origine de sa thèse de la « dualité du dynamisme humain » (1854). Lordat ira jusqu'à placer derrière l'âme immatérielle, une « âme de seconde majesté » présidant aux phénomènes vitaux (Dulieu, 1983). C'est là une traduction très connotée, tant sur le plan politique que religieux, du « principe vital» de Barthez. L'oeuvre de Lordat témoigne d'une collusion, qui reste exceptionnelle, des questions métaphysiques et doctrinales.

Toutefois, la crispation tardive de l'école de Montpellier sur une philosophie de nature spiritualiste n'est pas en mesure d'expliquer le fondement de la controverse. Celle-ci avait pris son essor au temps de Barthez, c'est-à-dire bien avant que la dérive spiritualiste ne vienne se greffer sur le vitalisme.

\subsection{Le facteur productif}

Focalisons-nous maintenant sur les publications des agents qui prirent part à la polémique. On peut estimer le rapport des forces entre les écoles, en comparant le nombre de textes affiliés aux doctrines de Montpellier ou de Paris ${ }^{6}$. On obtient ainsi un mesure approchée: 1) de la pr o-

\footnotetext{
${ }^{6}$ La liste en a été établie à partir du Catalogue général de la Bibliothèque nationale . Nous avons, pour des raisons volumétr i-
} 
ductivité scientifique, en prenant en compte les premières publications (colonne p); 2) du crédit scientifique, en comptant les rééditions (colonne r); 3) du volume global d'activité, en cumulant publications et rééditions (colonne t) (Tableau 5). Si l'on procède au décompte, on constate que la productivité parisienne est supérieure à celle de Montpellier (77 > 43), que le crédit accordé aux thèses parisiennes est supérieur à celles de Montpellier $(57>15)$, et que le volume total des publications témoigne de l'écrasante supériorité de l'école parisienne (134 > 58).

\begin{tabular}{|c|c|c|c|c|c|c|c|c|c|}
\hline \multirow[b]{2}{*}{ Auteur } & \multicolumn{3}{|c|}{ vitalistes } & \multicolumn{3}{|c|}{ organo-vitalistes } & \multicolumn{3}{|c|}{ organicistes } \\
\hline & $p$ & $r$ & $t$ & $p$ & $r$ & $t$ & $p$ & $r$ & $t$ \\
\hline Barthez & 7 & 9 & 16 & & & & & & \\
\hline Dumas & 6 & 3 & 9 & & & & & & \\
\hline Lordat & 15 & 0 & 15 & & & & & & \\
\hline Bérard & 7 & 2 & 9 & & & & & & \\
\hline Alquié & 8 & 1 & 9 & & & & & & \\
\hline Bichat & & & & 5 & 19 & 24 & & & \\
\hline Dugès & & & & 4 & 0 & 4 & & & \\
\hline Corvisart & & & & & & & 4 & 2 & 6 \\
\hline Broussais & & & & & & & 20 & 18 & 38 \\
\hline Laënnec & & & & & & & 5 & 6 & 11 \\
\hline Magendie & & & & & & & 25 & 14 & 39 \\
\hline Bernard & & & & & & & 23 & 17 & 40 \\
\hline Total & 43 & 15 & 58 & 9 & 19 & 28 & 77 & 57 & 134 \\
\hline
\end{tabular}

\section{Tableau 5: Crédit des écoles en nombre de publications}

La mesure de l'activité scientifique des deux écoles peut être précisée en ventilant, décennie par décennie, les publications respectives des agents qui prirent part à la controverse (Tableau 6).

Tableau 6: Histogramme des publications médicales (1790-1870)

ques, supprimé de la version définitive de cet article le détail des notices bibliographiques. Cette statistique ne tient compte que des oeuvres médicales, à l'exclusion des discours d'inauguration et des mémoires historiques sans exposé doctrinal. 
On observe que les succès de la médecine d'observation furent acquis dans les années 18201830, et que la riposte tardive de Montpellier (1850-1860) ne suffit pas à enrayer le développement de la doctrine parisienne.

\subsection{Le facteur institutionnel}

Comme le constate Schweber dans une critique des travaux se réclamant de la nouvelle sociologie des sciences: «Un second problème plus sérieux concerne la quasi-absence de considérations institutionnelles dans la plupart des études de la SSK... Les études sur les controverses, par exemple, [passent] directement des intérêts techniques aux intérêts idéologiques et sociaux, négligeant l'influence des facteurs comme les organisations formelles... » (1997: 89). Le rôle des facteurs institutionnels n'en mérite qu'un examen plus précis. On observe sans difficulté que les deux approches médicales se diffusèrent dans des institutions et des réseaux scientifiques distincts. Deux indicateurs permettent de s'en assurer: le statut des différents agents de la controverse à l'Académie des Sciences (titulaire, adjoint, associé, correspondant); leur appartenance à une institution universitaire ou extra-universitaire.

Mis à part Broussais, qui fut élu à l'Académie des Sciences morales et politiques (1823), trois des cinq Parisiens furent membres titulaires de l'Académie des Sciences: Corvisart (1811), Magendie (1821) et Claude Bernard (1854), lequel avait remporté trois fois le prix de physiologie pour ses travaux sur le système nerveux et le grand sympathique. Montpellier ne comptait aucun membre titulaire de l'Académie des Sciences. Barthez et Dumas étaient membres associés (1782, 1800), Dugès membre correspondant (1836). Cette dissymétrie établit par une autre voie que la thèse barthézienne jouissait, au temps de cette controverse, d'une moindre crédibilité scientifique. Mais, du point de vue relativiste, cette dissymétrie affectant l'estime scientifique pourrait être interprétée comme le fait d'une plus grande facilité d'accès des Parisiens à l'Institut.

Quant au partage entre les institutions universitaires et extra-universitaires, on constate que la doctrine vitaliste se développa presque exclusivement au sein des facultés de médecine (passées écoles de santé de 1794 à 1806). Les Montpelliérains furent tous des universitaires. Sous l'Ancien Régime, Barthez avait occupé la chaire d'Anatomie et de Botanique (1761). Oublié de la création 
de l'Ecole de santé — pour des raisons que nous examinerons plus loin — il sera nommé professeur honoraire en nivôse an IX (1800). Dumas eut en revanche une chaire d'Anatomie et Physiologie dès la création de l'école (1794). Il occupera plus tard la chaire de Clinique interne (1809). Lordat prit la chaire de Clinique opérante et cas rares (1811) avant d'être muté, à sa convenance, sur celle d'Anatomie et Physiologie (1813). Celle-ci deviendra en 1824 la chaire de Physiologie humaine par scission avec celle d'Anatomie. À Montpellier, Bérard obtint la chaire de Thérapeutique et matière médicale (1827); Alquié celle de Clinique externe (1850).

À la différence, la doctrine organiciste se développa moins au sein de la faculté que dans des institutions extra-universitaires de la capitale. Corvisart, après avoir été professeur de Clinique interne à l'Hôpital de la Charité, eut la chaire de Médecine du Collège de France (1797). Laënnec, sorti de l'hôpital Necker, prit la chaire de Corvisart au Collège de France (1822). Magendie fut également professeur de Médecine expérimentale au Collège de France (1830-1855). Claude Bernard, l'ancien préparateur de Magendie, héritera de la chaire de son devancier à la mort de celui-ci (1855). Notons d'ailleurs que Bernard ne quittera le Collège de France que pour enseigner la Physiologie comparée au Muséum d'histoire naturelle (1868), qui était aussi une institution autonome.

Alors que tous les Barthéziens étaient des universitaires, quatre des cinq agents parisiens engagés dans la controverse enseignaient au-dehors de l'Université: les quatre au Collège de France, un au Muséum d'histoire naturelle (le cas de Broussais est atypique). Or, il existe une spécificité de ces deux institutions. Par vocation, l'attachement doctrinal y est moins fort qu'au sein de l'Université, parce que ces institutions, certes anciennes ${ }^{7}$, ont moins pour fonction de soumettre l'auditeur à l'autorité de quelque maître, que de donner la possibilité aux chercheurs de dispenser un enseignement en rapport direct avec leurs découvertes récentes. À l'opposé, le statut des écoles de santé ne les destinait pas à être un lieu de recherche. Elles furent tournées, dès leur création, vers la pratique médicale. Comme le rappelle Ackerknecht: «L'Ecole de santé se voulait une école spécialisée, destinée essentiellement à former des praticiens. Les scientifiques étaient relégués soi-

\footnotetext{
${ }^{7}$ Le Collège royal, fondé en 1530 par François Ier, prit successivement les noms de Collège national et de Collège impérial, avant de devenir le Collège de France sous la Restauration. Le Jardin des herbes médicinales, fondé par Louis XIII en 1635, deviendra le Muséum d'histoire naturelle en 1794, sur ordre de la Convention. Les deux insitutions sont restées indépendantes de l'Université.
} 
gneusement à l'écart, dans des « réserves » telles que le Muséum et le Collège de France, qui étaient des institutions destinées essentiellement à la recherche. » (1986: 53).

On observe ici une correspondance étroite du partage des institutions scientifiques qui accueillirent les agents de la controverse avec celui que nous avons pu constater sur les méthodes, les doctrines et la productivité scientifique. Cette correspondance demande une explication.

\subsection{Le facteur professionnel}

Après le décret de l'an I, portant suppression des universités, la Convention procéda, par un nouveau décret du 14 frimaire an III (4 décembre 1794), à la création de trois écoles de santé: Paris, Montpellier, Strasbourg. Ce décret était assorti d'une mesure fixant les effectifs consentis aux trois écoles: 300 à Paris, 150 à Montpellier et 100 à Strasbourg. On peut, en comparant ces chiffres avec ceux du Tableau 2, calculer le taux de variation des effectifs, de l'Ancien Régime à la Convention (Tableau 7):

\begin{tabular}{lrrr} 
Ecole de santé & avant 1789 & après 1794 & variation \\
\hline Paris & $29,9 \%$ & $54,5 \%$ & $+82 \%$ \\
Montpellier & $46,7 \%$ & $27,3 \%$ & $-41 \%$ \\
Strasbourg & $23,4 \%$ & $18,2 \%$ & $-22 \%$ \\
Total & $100,0 \%$ & $100,0 \%$ & -
\end{tabular}

Tableau 7: Effectifs comparés des trois écoles de santé

Montpellier accuse la variation négative la plus forte $(-41 \%)$, alors que les effectifs relatifs de Paris sont en augmentation de $+82 \%$. Marque claire du centralisme impulsé par les Montagnards, cette mesure imposa une hiérarchie entre les écoles et scella le déclin de l'école de Montpellier, désormais condamnée à se satisfaire d'une audience régionale. Lésée dans ses intérêts, l'école de Montpellier avait toutes les raisons de nourrir de la rancoeur contre le pouvoir qui avait mis un terme à son développement. Les intérêts professionnels furent donc de la partie, et ils occasionnèrent une dispute secondaire sur les valeurs du centralisme et du régionalisme, ainsi que sur l'opportunité qu'il avait à suivre aveuglément les ordres de la capitale.

Sur certains points, d'ailleurs, Montpellier eut raison de sa rivale. En 1814, le frère Elysée, 
chirurgien de Louis XVIII, tenta d'obtenir la séparation de la Chirurgie et de la Médecine et le rétablissement de la corporation des Chirurgiens-Barbiers. Alors que la faculté de médecine de Paris soutient la mesure, celle de Montpellier s'insurge contre un projet jugé, à juste titre, rétrograde. La querelle durera de 1814 à 1816, mais les raisons que Montpellier avait invoquées dans un mémoire adressé aux pouvoirs publics (1814) furent suivies. Cette réaction de Montpellier ne fut pas guidée par une position de principe, mais bien par ses intérêts professionnels. En effet, le frère Elysée avait pour objectif de rétablir la vente des brevets de lieutenance du Premier Chirurgien du Roi et les autorisations de remèdes, sources de revenus qui avaient été supprimées par l'organisation révolutionnaire. Montpellier s'opposa violemment à la réapparition de telles charges. La Chirurgie resta liée à la Médecine.

Mais si l'organisation d'une discipline scientifique est directement sujette aux intérêts professionnels de ses membres, il est plus difficile d'envisager pareille détermination dans la controverse qui nous occupe. Que Montpellier ait gardé rancoeur de sa rétrogradation de l'an III, et qu'elle en ait imaginé une crispation doctrinale, c'est possible. Mais on ne voit pas comment l'école aurait pu concevoir - a priori - d'adopter l'anti-doctrine de Paris, car c'est là, précisément, qu'elle aurait menacé ses intérêts professionnels, en prenant le risque de perdre la face aux yeux de la communauté médicale. L'humiliation initiale de Montpellier explique sans doute le choix d'une doctrine médicale différente de celle de Paris, mais ne rend pas compte du fait que celle-ci ait été le vitalisme.

\subsection{Le facteur politique}

Ackerknecht (1986) a montré que les principes d'une médecine d'observation et d'expérimentation - dont on a vu qu'ils étaient au coeur de l'opposition entre Paris et Montpellier étaient inscrits dans la création des écoles de santé. Ce fut une décision politique, comme en atteste le rapport de Fourcroy à la Convention du 27 novembre 1794:

«Dans l'Ecole centrale de santé... la pratique, la manipulation seront jointes aux préceptes théoriques. Les élèves seront exercés aux expériences cliniques, aux dissections anatomiques, aux opérations chirurgicales, aux appareils. Peu lire, beaucoup voir et beaucoup faire, telle sera la base du nouvel enseignement que les Comités vous proposent de décréter. » (cf. Ackerknecht, 1986: 47). 
Ce texte témoigne donc de l'existence éventuelle de rapports, entre les facteurs institutionnel et politique, qui doivent être interrogés. On peut en effet se demander si les agents qui ont adhéré ou rejeté les principes de la médecine expérimentale n'ont pas agi davantage par conviction politique que par détermination scientifique. Avant d'affirmer de façon péremtoire qu'il y a eu débat rationnel, comme le voudraient les adeptes du rationalisme, il convient au préalable de s'assurer que les médecins parisiens n'ont pas été plus sensibles à l'esprit révolutionnaire que ne le furent les Montpelliérains.

Une première indication en ce sens nous est offerte par le chef de file du vitalisme. On sait en effet que Barthez nourrissait une hostilité vis-à-vis des idéaux révolutionnaires. À l'ouverture des Etats généraux, il fit paraître un opuscule intitulé: Libre discours sur la prérogative que doit avoir la noblesse dans la Constitution et les Etats généraux de la France (1789). Ce texte fut sans doute motivé par le fait que Barthez était, depuis 1781, médecin ordinaire du duc d'Orléans. Lorsque les événements de 1789 éclatèrent, Barthez, se jugeant compromis, prit la fuite pour Narbonne et démissionna de ses fonctions de professeur de l'école de Montpellier le 2 thermidor an II (1793). Son regain d'audience de 1802 ne s'explique que par la bienveillance de Chaptal (lequel était issu de Montpellier).

Mais peut-on pour autant affirmer que tous les Montpelliérains furent des royalistes? Dumas, qui fut l'un des premiers à prendre le relais des thèses vitalistes de Barthez, échappe à cette détermination. Professeur à l'école centrale de Lyon en 1793, il s'afficha en septembre dans une Assemblée du peuple demandant la capitulation de la ville. Les royalistes lui en tinrent rigueur et le firent emprisonner. Ayant échappé à l'échafaud de justesse, il obtint de Paris une mutation vers Nice, dans la future armée d'Italie. De retour à Montpellier, il s'essaya de nouveau à la politique aux côtés de Cambacérès (un modéré, qui fut néanmoins exilé sous la Restauration). Quant à Dumas, ses actes politiques lui valurent d'être le premier directeur de l'école de santé de Montpellier (1794) et, pour peu de temps, Président de la municipalité (1798-1799).

Bérard montre une accointance assez nuancée avec les structures politiques de la Restauration. Une des décisions que prirent les Bourbons à leur retour fut de supprimer le concours professoral dans les écoles de médecine. Les professeurs seraient nommés par Paris. Or, cependant que Prunelle et Broussonnet sont cassés de leurs fonctions en raison de leurs antécédents politiques, Bérard obtient une chaire de Mgr de Frayssinous, alors Ministre de l'Instruction publique et 
Grand Maître de l'Université (1823). Il est difficile de déterminer si Bérard fit alliance par conviction politique ou par opportunisme. Cette ambiguïté est encore accentuée par la lecture de son Mémoire sur les avantages scientifiques et politiques du concours (1820), rédigé cinq ans après les débuts de la Restauration, et dans lequel Bérard défend une position aux antipodes de celle adoptée par les Bourbons.

Lordat, défenseur des thèses de l'école de médecine de Montpellier, ne suivra Barthez en politique qu'assez tardivement. Il faut en effet se rappeler que, parti pour Paris en 1793, le jeune Lordat adhéra à la nouvelle Constitution, avant de devenir chirurgien militaire (15 germinal an II). C'est bien après la rencontre avec Barthez (1806) qu'il défendit une articulation du vitalisme avec les idées conservatrices de Louis de Bonald (1754-1840). Aux alentours de 1850, le vitalisme était agonisant sur le plan scientifique, et Jacques Lordat crut bon de proposer un « accord » entre sa conception philosophico-médicale - déjà fortement teintée de spiritualisme - et la doctrine de Bonald, chef de file des ultras. On doit à ce successeur de Barthez deux textes dans la même veine: Accord de la doctrine de Montpellier avec ce que demandent les lois, la morale publique et les enseignements religieux (1852) et Apologie de la définition bonaldienne de l'homme (1854), textes qui trahissent des convictions monarchistes et traditionalistes marquées. Lordat était alors un vieillard de 81 ans.

On sait peu de choses sur les opinions politiques d'Alexis Alquié, sinon qu'il fit partie de la commission de 1852, chargée de prêter allégeance au Second Empire, de manière à sauvegarder les intérêts de la faculté. Cet acte a, en soi, une signification politique très limitée. On sait en effet que tout au long du XIXe siècle les professeurs durent prêter serment, tantôt à la République, tantôt à l'Empire. La déclaration d'allégeance au dernier régime en place était devenu un acte de routine administrative. La demande formulée par la commission à cette occasion ne sera d'ailleurs pas entendue: Napoléon III supprimera à nouveau le concours professoral.

Ge portrait nuancé pourrait être répété, trait pour trait, sur les agents parisiens. On y trouve des révolutionnaires, des royalistes et beaucoup de radicaux démocrates, qui tentent de rénover les conceptions médicales à l'écart des vicissitudes politiques du moment.

Broussais est sans doute le médecin français dont l'engagement dans les événements de la Révolution est le plus marqué. C'était un libéral bonapartiste. Breton de naissance, Broussais combattit les Chouans dans les rangs de l'armée révolutionnaire (1792-1794). Par mesure de représailles, ses parents furent assassinés à Noël 1795. Broussais suivit les armées napoléoniennes et 
fit campagne en Hollande, en Allemagne, en Autriche en Italie et en Espagne. En 1828, alors que l'Université était dominée par les Bourbons et les Jésuites, il fit paraître un texte polémique sur les attaques des « Kanto-Platoniciens » - autrement dit les spiritualistes et autres éclectiques — qu'il ne manque pas de qualifier au passage de « lécheurs de bottes des Prussiens ». On trouve ici l'association, proposée par un médecin de l'époque, entre un ensemble de doctrines médicales (au nombre desquelles le vitalisme) et une conception politique. Mais cette association ne doit pas faire illusion. Broussais était un bon tacticien, et jouait facilement de la corde sensible pour s'attirer les faveurs du public (Ackerknecht, 1986: 82-83). Il serait donc douteux de conclure à une détermination pure et simple des contenus scientifiques par les idées politiques. Broussais fait un calcul de rationalité subjective: il mobilise le spectre prussien pour tenter de remplir des amphithéâtres qui, à l'époque, ne cessaient de se vider.

On a peu d'indications sur les accointances politiques de Corvisart. Au tout début de sa carrière, c'est-à-dire sous l'Ancien Régime, il s'était présenté pour être médecin à l'Hôpital Necker. Il en fut écarté pour un motif qui peut avoir une signification politique: Corvisart refusait de porter perruque. Un autre indice est qu'il fut nommé professeur dès 1794, à la refonte de l'organisation médicale. Un royaliste affiché aurait du attendre la Restauration pour se voir attribuer une charge de professeur. En outre, le disciple qui était devenu son adjoint, Leroux des Tillets, fut limogé en 1822 par les Bourbons.

Au contraire, Laënnec garda sa vie durant de profondes attaches jésuites et royalistes, ce qui lui valut d'ailleurs les violentes invectives de Broussais. À Paris, Laënnec fréquentait le cercle des Récamier, Martinet, Antoine-Laurent Bayle, Cayol et Chomel qui étaient tous des réactionnaires et des orléanistes. Mais il est difficile d'établir une relation directe entre les convictions intimes de Laënnec et ses options scientifiques. Comme le dit Ackerknecht, « ce caractère traditionaliste se reflète dans ses convictions politico-religieuses, mais est en totale contradiction avec tout ce qu'il a apporté de novateur en médecine » (1986: 130).

L'examen du facteur politique montre que les initiateurs de la médecine organiciste comptaient un libéral (Broussais), un révolutionnaire modéré (Corvisart) et un jésuite royaliste (Laënnec). Les thèses vitalistes furent défendues par un monarchiste (Barthez), un révolutionnaire (Dumas), et un homme qui, devenu octogénaire, afficha des idées réactionnaires (Lordat), les autres agents de la controverse n'ayant pas de conviction saillante. Cette dispersion des opinions politi- 
ques peut être rapprochée du résultat auquel parvient Schweber dans une étude de la statistique au XIX ${ }^{\circ}$ siècle: «En France, les débats de 1837 et de 1867 furent caractérisés par une disjonction entre les différents types d'arguments... En d'autres termes, les positions des individus sur des questions techniques ne permettent pas de prévoir leurs positions sur des questions politiques ou professionnelles. » (1997: 85).

\section{Conclusions}

Si l'on accepte d'analyser la controverse entre les écoles de médecine de Paris et de Montpellier en considérant que les facteurs étudiés jouissent d'une relative indépendance les uns vis-à-vis des autres, on peut affirmer que seuls quatre facteurs coïncident étroitement avec l'opposition des écoles (méthode, doctrine, productivité scientifique et institution). Les trois autres facteurs (philosophique, professionnel et politique) ont peut-être orienté la crispation de l'école de Montpellier sur une doctrine différente de celle enseignée à Paris, mais ils n'ont pas influencé le contenu de cette doctrine. Il reste à voir pourquoi les études relativistes tendent généralement à assurer ce type de résultat.

\subsection{La notion de " contenu scientifique»}

Cette controverse offre une singularité historique qui se soumet avec difficulté aux exigences du programme relativiste, qui prétend que la stabilisation d'un énoncé scientifique résulte de la négociation sociale à laquelle il donne lieu. Cet article laisse entrevoir une conclusion assez différente: 1) La controverse a été réglée à la faveur de ceux qui ont délaissé la spéculation philosophique pour pratiquer des observations et des expériences systématiques; 2) La doctrine de l'école de Montpellier s'est effacée devant sa rivale parce que les Parisiens avaient une productivité scientifique nettement supérieure; 3) La suprématie de l'école de Paris a été favorisée par des institutions aptes à accueillir ses recherches novatrices (Collège de France, Muséum).

Le facteur institutionnel - qui est, stricto sensu, le seul facteur extra-scientifique mêlé à la controverse - n'est pas un argument en faveur du relativisme. On s'en rendra compte en posant la question: Peut-on incriminer le facteur institutionnel pour expliquer le succès de l'école de $\mathrm{Pa}$ - 
ris? Contre toute attente, en effet, la réponse ne peut être que négative. Commençons par une observation relative au «mode de rejet» (Collins \& Pinch, 1991: 302). Lorsque des connaissances A ne sont pas testables — c'est le cas du « principe vital » ou du « génie périodique pernicieux » - il est peu valorisant pour un partisan de B, qui produit des connaissances testables, de se lancer dans une réfutation de la théorie A. Les moyens étant disproportionnés par rapport aux fins, il a toutes les chances de pratiquer un « rejet implicite » de A. Le règlement de la controverse ne procède alors pas d'une invalidation de la thèse $\mathrm{A}$, mais du seul niveau de productivité de $\mathrm{B}$. Il est raisonnable de penser, à la suite de Ben-David (1994), que la productivité scientifique est déter-

minée par les formes d'organisation de la recherche. À la différence de l'Université, les institutions indépendantes qu'étaient le Collège de France et le Muséum réunissaient les conditions pour favoriser l'essor de la médecine expérimentale. L'équipement des laboratoires y était plus conséquent; la spécialisation valorisée; les tâches d'enseignement moins contraignantes. Les chercheurs pouvaient donc s'y consacrer plus librement à la recherche.

Les analyses de Ben-David (1994) ne doivent pas pour autant être extrapolées aux contenus scientifiques. En effet, si l'auteur constate que certaines formes d'organisation sont, plus que d'autres, aptes à augmenter la productivité scientifique des chercheurs, il ne rend pas ces formes responsables du succès ou de l'échec des théories scientifiques. Comme le reconnaît Gad Freudenthal dans sa préface: « Ben-David ne porte pas atteinte à l'autonomie des sciences, ni explicitement, ni implicitement: pour lui, les conditions sociales peuvent déterminer le "comment" et le "quand" de l'émergence de nouvelles disciplines ou théories scientifiques et elles déterminent également la vitesse de leur développement, mais elles n'interviennent pas dans la validation empirique des propositions scientifiques... Ben-David s'oppose à toute dérive relativiste... » (Ben-David, 1994: 2). Dans la controverse étudiée, le facteur extra-scientifique « institutionnel » a déterminé, non pas les contenus, mais la productivité des chercheurs, ce qui incite à clairement distinguer ces notions.

\subsection{La notion de « détermination»}

Les résultats auxquels nous parvenons sont donc cohérents avec le rationalisme qui assujettit les contenus scientifiques au seul critère de la preuve. Ils incitent à rejeter l'idée d'une détermination causale des contenus prônée par le programme fort de Bloor (1976) et la position de principe de 
Shapin et Schaffer (1993) qui entendent mettre en évidence le caractère politique de toute connaissance. Selon eux, il conviendrait de retenir de la controverse entre Hobbes et Boyle, que « l'issue de la lutte entre... les productions intellectuelles dépend du succès politique de la tentative des diverses candidates à se mêler aux activités d'autres institutions et d'autres groupes d'intérêts. Gagne celle qui possède le plus d'alliés et les alliés les plus puissants. » (1993: 342-343).

La controverse que nous venons d'étudier suggère une conclusion très différente, en particulier parce que la division politique des agents ne correspond pas au partage des contenus scientifiques. La position de Collins (1981) est plus admissible, dans la mesure où la relation de causalité efficiente laisse place à un rapport plus large. Mais Collins omet de préciser le sens de ce rapport et les sphères qu'il implique: il s'agirait simplement des «structures politiques et sociales ». L'étude de la controverse entre les écoles de Paris et de Montpellier permet de préciser ces éléments. Elle montre que l'activité scientifique dépend du facteur extra-scientifique qui est le plus étroitement lié à elle, c'est-à-dire les formes d'organisation au sein desquelles s'effectue la recherche; et que son influence s'exerce sur la productivité. Plus explicitement, cela signifie que l'« esprit » qui régnait au Collège de France - c'est-à-dire les normes de comportement qui y étaient admises — a permis l'apparition de contenus scientifiques nouveaux et, à ce titre seulement, opposés au vitalisme de l'école de Montpellier. Cette relation avec un facteur externe ne signifie donc pas que l'on soit en mesure, étant donné un facteur extra-scientifique, de prédire le contenu de la théorie soumise à ce facteur. Ce rapport n'est pas une relation causale ou fonctionnelle. Il appartient à la classe des relations signifiantes (Merton, 1973: 12). Le concept de congruence, restreint au sens d'une adéquation des contenus scientifiques avec des valeurs et des normes du comportement scientifique, est sans doute celui qui offre le plus de pertinence sociologique pour comprendre ce type de rapport. Ces précisions rabaissent la portée des analyses relativistes qui n'ont pas su distinguer les multiples formes de relation entre les contenus scientifiques et l'environnement qui les produit: il n'y a aucune raison de confondre une détermination causale ou fonctionnelle avec une correspondance ou un effet de la congruence.

Il existe enfin deux arguments qui s'opposent formellement à l'idée d'une détermination des contenus en jeu dans la controverse étudiée.

1) Supposons, sous la clause ceteris paribus, que le vitalisme ait été défendu à Paris et non à Montpellier. La thèse d'une détermination des contenus par des facteurs extra-scientifiques prédit 
que l'école de Montpellier aurait dû défendre l'organicisme (puisque la rancoeur provoquée par le décret de l'an III, les intérêts professionnels et les différences institutionnelles auraient été identiquement répartis). On objectera que cette reconstruction rationnelle est peut-être une fantaisie ${ }^{8}$, mais elle montre une limite du programme relativiste. Celui-ci n'est pas en mesure d'expliquer pourquoi - sous la clause ceteris paribus - l'école de Montpellier n'aurait pas continué d'adhérer au vitalisme, en nourrissant par ailleurs une querelle avec Paris à propos des effectifs étudiants ou de l'organisation de la profession... Pourquoi une querelle politique ou professionnelle devrait-elle nécessairement se répercuter sur les contenus scientifiques? L'hypothèse d'un tel rapport a maintes fois été suggérée par les relativistes, mais sa preuve n'a jamais été apportée.

2) Supposons maintenant que Paris ait soutenu une doctrine organiciste, mais que nous ne connaissions pas la doctrine de Montpellier. Les facteurs extra-scientifiques, pris isolément, ne sont pas davantage en mesure de prescrire a priori le contenu vitaliste de la doctrine de Montpellier. Il n'existe aucune raison valable pour penser que l'école de Montpellier aurait du défendre le vitalisme plutôt que n'importe laquelle des thèses médicales du XVIIIe siècle échappant un tant soit peu aux canons de la nouvelle médecine - disons, pour fixer les idées, l'animisme de Stahl ou l'humoralisme de Friedrich Hoffmann. Si les relativistes devaient répondre que Montpellier a adopté le vitalisme parce que Barthez l'avait enseigné dans cette faculté, ils devraient alors — par simple application d'un principe de symétrie - expliquer pourquoi la médecine parisienne, qui comptait pourtant des Chaussier, Pinel et Bichat, ouverts au vitalisme, se détourna massivement de cette doctrine spéculative pour se lancer dans un programme d'expérimentation. C'est là une deuxième limite du relativisme, imposée par ses aspects conventionnalistes.

Ces insuffisances incitent en définitive à envisager très sérieusement une approche rationaliste des contenus scientifiques. Il faut rappeler que cette approche admet sans difficulté que les connaissances scientifiques soient le résultat d'une « construction ", mais en fait une lecture très différente de celle que propose le relativisme. Comme l'a montré Boudon (1994: 22) le fait qu'il y ait une discussion des contenus entre chercheurs ne signifie pas ipso facto que le règlement de la

\footnotetext{
${ }^{8}$ Cette fantaisie suit cependant très exactement les idées de Weber sur la possibilité objective et l'usage de tableaux idéaux (Phantasiebilder). Comme il l'écrit: « Il n'y a absolument rien de "oiseux" à poser la question: qu'aurait-il pu
} 
discussion procède d'une négociation sociale (au sens où la vérité serait établie par une sorte de « compromis » entre les chercheurs). On peut interpréter cette construction, en disant qu'une discussion scientifique s'éteint quand des preuves rationnelles suffisantes ont été avancées et comprises des contradicteurs. La durée d'extinction d'une controverse peut également être interprée dans le cadre rationaliste. Lakatos (1994) a montré que les chercheurs abandonnent rarement une théorie contredite par une expérience isolée. Mais il n'en fait pas pour autant la marque d'un attachement arbitraire aux contenus. Il explique cette inertie, propre au règlement de toute controverse, par la fonction qu'exerce le « glacis protecteur » (protective belt) sur le « noyau dur » (hard core) de la théorie.

Dans le débat entre le relativisme et le rationalisme, ce n'est donc pas la possibilité d'une étude sociologique des contenus qui doit être aujourd'hui remise en question, mais l'a priori relativiste qui sous-tend en général ce genre de tentatives. En définitive, cet article renforce la possibilité d'un programme rationaliste en sociologie de la connaissance scientifique.

\section{Bibliographie}

Ackerknecht E.H., 1986. - La Médecine hospitalière à Paris (1794-1848), Paris, Payot.

Ben-David J., 1994. - Eléments d'une sociologie historique des sciences, Paris, Presses universitaires de France.

Bérard F., 1819. - Observations cliniques pour servir de preuve à la doctrine médicale de l'école de Montpellier, Montpellier, Imprimerie J. Martel.

Bloor D., 1983. - Sociologie de la logique ou les limites de l'épistémologie, Paris, Pandore [1976].

Boudon R. \& Clavelin M. éds., 1994. - Le Relativisme est-il résistible? Regards sur la sociologie des sciences, Paris, PUF.

Brockliss L.W., 1992. - La querelle entre les facultés de médecine de Montpellier et de Paris au XVIIe siècle, VIIe Centenaire des Universités de l'Académie de Montpellier (1289-1989), Université de Montpellier I, pp. 44-49.

Callon M. \& Latour B. dirs., 1991. - La science telle qu'elle se fait, Paris, Editions La Découverte.

Chrestien A., 1860. - Lettre à son Exc. M. Rouland, Ministre de l'Instruction publique et des Cultes, sur l'antagonisme qui a toujours existé entre la faculté de médecine de Montpellier et celle de Paris, Cette, Typographie G. Bonnet.

Collins H., 1981. - Stages in the Empirical Programme of Relativism, Social Studies of Science, 11 (1), pp. 3-11.

Collins H. \& Pinch T., 1991. - En parapsychologie, rien ne se passe qui ne soit scientifique, Callon M. \& Latour B. eds., La Science telle qu'elle se fait, Paris, Editions La Découverte, pp. 297-343.

Collins H. \& Pinch T., 1994. - Tout ce que vous devriez savoir sur la science, Paris, Le Seuil [1993].

Dulieu L., 1983 -1990. - La Médecine à Montpellier, III: L'Âge classique, 1: 1983, 2: 1986. IV: De la Première à la Troisième

arriver si Bismarck n'avait pas pris la décision de faire la guerre? » (Weber, 1964: 270). 
République, 1: 1988, 2: 1990, Avignon, Les Presses universelles.

Dulieu L. dir., 1990. - La Médecine à Montpellier du XIIe au XXe siècle (30e Congrès international d'histoire de la médecine, septembre 1986), Paris, Hervas.

Garrison F.H. 1929. - An Introduction to the History of Medicine, Philadelphia / London, Saunders.

Goubert J.-P. \& Rey R. éds., 1993. - Atlas de la Révolution française, t. 7: Médecine et santé, Paris, Editions de l'EHESS.

Isambert F.-A., 1985. - Un « programme fort » en sociologie de la science? Revue française de sociologie, 26, pp. 481-508.

Isambert F.-A., 1994. - Après l'échec du «programme fort », une sociologie du contenu de la science reste-t-elle possible? Boudon, R. \& Clavelin, M. éds., Le Relativisme est-il résistible? Regards sur la sociologie des sciences, Paris, PUF, pp. 51-76.

Julia D. \& Revel J., 1989. - Les Universités européennes du XVIe au XVIIIe siècle, Histoire sociale des populations étudiantes, tome 2. Paris, Editions de l'EHESS.

Julia D., 1992. - L'université de médecine de Montpellier à l'époque moderne, VIIe Centenaire des Universités de l'Académie de Montpellier (1289-1989), Montpellier, Université de Montpellier I, pp. 26-38.

Kühnholtz H., 1843. - Paris et Montpellier sous le rapport de la philosophie médicale... Paris, J.-B. Baillière \& al., Montpellier, L. Castel Libraire-éditeur.

Lakatos I., 1994. - Histoire et méthodologie des sciences, Paris, Presses universitaires de France.

Latour B. \& Woolgar S., 1988. - La Vie de laboratoire. La production des faits scientifiques, Paris, La Découverte [1979].

Latour B., 1989. - Pasteur et Pouchet: hétérogenèse de l'histoire des sciences, M. Serres, dir., Eléments d'histoire des sciences, Paris, Bordas, pp. 423-445.

Lavabre-Bertrand T., 1992. - La philosophie médicale de l'école de Montpellier au XVIIIe et XIXe siècles, VIIe Centenaire des Universités de l'Académie de Montpellier (1289-1989), Montpellier, Université de Montpellier I, pp. 6369.

Lécuyer B.-P. \& al., 1988. - Sociologie des sciences et des techniques, L'Année Sociologique, 38, pp. 381-411.

Lordat J., 1842. - Apologie de l'Ecole médicale de Montpellier, en réponse à la lettre écrite par M. Peisse à M. le Professeur Lordat, Paris/Montpellier, J.-B. Baillière et L. Castel Libraire.

Merton R.K., 1973. - The Sociology of science. Theoretical and empirical investigations, edited with an introduction by Norman W. Storer, Chicago/London, The University of Chicago.

Moussy H., 1992. - L'Ecole de santé de Montpellier, 1794-1803, VIIe Centenaire des Universités de l'Académie de Montpellier (1289-1989), Montpellier, Université de Montpellier I, pp. 59-62.

Schweber L., 1997. - Controverses et styles de raisonnement, Enquête, 5, pp. 83-108.

Shapin S. et Schaffer S., 1993. - Léviathan et la Pompe à air, Hobbes et Boyle entre science et politique, Paris, La Découverte.

Vidal Y., 1958. - La Bibliothèque et les Archives de la faculté de médecine de Montpellier, Montpellier, Causse, Graille \& Castelnau éditeurs.

Weber M., 1964. - Possibilité objective et causalité adéquate en histoire, Essais sur la théorie de la science, Paris, Plon, pp. 269-299 [1906]. 